\title{
High-scale inflation and the tensor tilt
}

\author{
Daniel Baumann, Hayden Lee and Guilherme L. Pimentel \\ Department of Applied Mathematics and Theoretical Physics, Cambridge University, \\ Wilberforce Road, Cambridge, CB3 0WA, U.K. \\ E-mail: D.Baumann@damtp.cam.ac.uk, h.lee@damtp.cam.ac.uk, \\ gp403@cam.ac .uk
}

ABSTRACT: In this paper, we explore a novel observational signature of gravitational corrections during slow-roll inflation. We study the coupling of the inflaton field to highercurvature tensors in models with a minimal breaking of conformal symmetry. In that case, the most general correction to the tensor two-point function is captured by a coupling to the square of the Weyl tensor. We show that these scenarios lead to a correction to the tilt of the tensor power spectrum and hence a violation of the tensor consistency condition. We arrive at the same conclusion through an analysis in conformal perturbation theory.

KEYwords: Models of Quantum Gravity, Conformal and W Symmetry, Space-Time Symmetries, Gauge-gravity correspondence

ArXiv EPrint: 1507.07250 


\section{Contents}

1 Introduction 1

2 Tensors beyond Einstein gravity 3

2.1 Weakly broken conformal symmetry 3

2.2 Violation of the consistency condition 5

$\begin{array}{llr}3 & \text { CFT interpretation } & 9\end{array}$

3.1 Wavefunction of the universe 9

$\begin{array}{ll}3.2 \text { Conformal perturbation theory } & 10\end{array}$

4 Conclusions $\quad 12$

$\begin{array}{ll}\text { A Comments on conformal symmetry } & 13\end{array}$

$\begin{array}{ll}\text { B Comments on Einstein frame } & 14\end{array}$

\section{Introduction}

String theory predicts higher-curvature corrections to the gravitational effective action. If the inflationary scale is sufficiently high, these corrections may be observable. In fact, the imprint of these effects on the primordial perturbations may be a rare observational window on inflationary models without a large hierarchy between the Hubble scale and the string scale. These models are hard to analyze, since, unlike more conventional inflationary theories, they cannot be organized as an expansion in a ratio of energy scales. To make robust statements therefore requires identifying observables which are protected by symmetries. In this paper, we will consider inflationary models whose predictions are controlled by the weakly broken conformal symmetry of the quasi-de Sitter background. Our approach is similar in spirit to that of [1] and [2] (see also [3-14]), where conformal symmetry was used to constrain the three-point functions for tensors and scalars, respectively. In particular, in [1], it was shown that higher-curvature corrections give rise to a new structure in the graviton three-point function. While the analysis of the tensor three-point function is particularly clean and model-insensitive, it is also hard to verify in observations, since tensor non-Gaussianities are likely to be very small. Here, we will discuss a potentially larger signature in the graviton two-point function.

The tensor power spectrum is characterized by an amplitude (or the tensor-to-scalar ratio, $r)$ and a tilt $\left(n_{t}\right)$. In single-field slow-roll inflation, minimally coupled to Einstein gravity, these two parameters are related by a consistency condition [15], $r=-8 n_{t}$. We will show that the leading higher-curvature corrections to the gravitational action lead to a 
violation of this consistency condition. ${ }^{1}$ We will arrive at this conclusion from two different perspectives:

- First, we consider a small breaking of de Sitter symmetry in the inflationary action, controlled by the slow-roll parameter $\varepsilon \equiv-\dot{H} / H^{2}$. In our model, conformal symmetry is broken by the inflaton potential and a coupling to the square of the Weyl tensor. These terms have been considered in the literature before, both from the perspective of an effective field theory [17] and in string theory [18] (see also [19-21]). We argue that this simple action reproduces the most general corrections to the tensor two-point function at leading order in the breaking of the conformal symmetry. While the tensor-to-scalar ratio is of order $\varepsilon$, the tensor tilt gets a correction of order $\sqrt{\varepsilon}(H / M)^{2}$, where $M$ is the scale suppressing the higher-curvature corrections. If the scale $M$ is close to the Hubble scale, this correction is the dominant effect. ${ }^{2}$

- Second, we analyze the same problem from the point of view of the wavefunction of the universe. The coefficients of the wavefunction are constrained by the de Sitter isometries, and can be interpreted as correlation functions of a putative conformal field theory (CFT) [22, 23]. To describe a realistic cosmology, we break the conformal symmetry by introducing a marginally relevant deformation of the CFT. If the deformation is small, then it can be treated in conformal perturbation theory [24]. The slow-roll parameter $\varepsilon$ is related to the coupling and the dimension of the operator that deforms the CFT [25]. We use conformal perturbation theory as a robust proof of our claim that the tensor-to-scalar ratio $r$ and the tensor tilt $n_{t}$ are non-zero at different orders in $\sqrt{\varepsilon}$. The advantage of this approach is that it relies mostly on symmetries, and allows us to be agnostic about the details of the effective action of the theory.

The outline of the paper is as follows. In section 2, we introduce a simple action in which higher-curvature corrections lead to a violation of the tensor consistency condition. We emphasize that the effect arises at leading order in the breaking of the de Sitter isometries. In section 3, we confirm this conclusion with an analysis of the stress tensor two-point function in a perturbed conformal field theory. Our conclusions are summarized in section 4. Two appendices contain technical details. In appendix A, we make a few remarks on the breaking of conformal symmetry in theories with a nontrivial sound speed. In appendix B, we review the argument of [26], showing that a nontrivial tensor spectrum can be mapped to a nontrivial scalar spectrum by a disformal transformation [27].

Notation and conventions. We will use natural units, $c=\hbar=1$, with reduced Planck mass $M_{\mathrm{pl}}^{2} \equiv 1 / 8 \pi G$. Our metric signature is $(-+++)$. Latin indices denote spatial coordinates (e.g. $x_{i}, k_{i}$ ), while Greek indices stand for spacetime coordinates. We will use both physical time $t$ and conformal time $\eta$. Overdots and primes will denote derivatives

\footnotetext{
${ }^{1}$ Related observations have appeared in [16].

${ }^{2}$ Corrections due to higher-derivative interactions of the inflaton, such as $(\partial \phi)^{4} / \Lambda^{4}$, will be suppressed by a larger scale $\Lambda$. As we will show, the hierarchy $\Lambda \gg M$ is protected because the gravitational coupling to the scalar sector is small.
} 
with respect to $t$ and $\eta$, respectively. The dimensionless power spectrum of a Fourier mode $f_{\vec{k}}$ is defined as

$$
\Delta_{f}^{2}(k) \equiv \frac{k^{3}}{2 \pi^{2}}\left\langle f_{\vec{k}} f_{-\vec{k}}\right\rangle^{\prime},
$$

where the prime on the correlation function indicates that the overall momentumconserving delta function is being dropped.

\section{Tensors beyond Einstein gravity}

In this section, we motivate a scenario in which the inflaton breaks the isometries of de Sitter space by a minimal amount, and analyze the consequences for the scalar and tensor two-point functions. We show that a coupling to the Weyl tensor leads to a violation of the tensor consistency condition.

\subsection{Weakly broken conformal symmetry}

De Sitter space is a solution to Einstein gravity with a positive cosmological constant

$$
S_{d S}=\frac{M_{\mathrm{pl}}^{2}}{2} \int \mathrm{d}^{4} x \sqrt{-g}(R-2 \Lambda) .
$$

In flat slicing, the line element is given by

$$
\mathrm{d} s^{2}=-\mathrm{d} t^{2}+e^{2 H t} \mathrm{~d} \vec{x}^{2}=\frac{-\mathrm{d} \eta^{2}+\mathrm{d} \vec{x}^{2}}{(H \eta)^{2}}, \quad \text { with } \quad 3 H^{2} \equiv \Lambda .
$$

Inspection of (2.2) reveals the isometries of the de Sitter spacetime: in addition to spatial rotations and translations, these include a dilatation and three special conformal transformations

$$
\begin{aligned}
\mathrm{D}: & \eta \rightarrow \eta(1+\lambda), & \vec{x} & \rightarrow \vec{x}(1+\lambda), \\
\mathrm{SCT}: & \eta \rightarrow \eta(1-2 \vec{b} \cdot \vec{x}), & \vec{x} & \rightarrow \vec{x}-2(\vec{b} \cdot \vec{x}) \vec{x}+\left(\vec{x}^{2}-\eta^{2}\right) \vec{b},
\end{aligned}
$$

where $\lambda$ and $\vec{b}$ are infinitesimal parameters. At late times, $\eta \rightarrow 0$, these isometries act as conformal transformations on the spacelike boundary $\mathcal{I}_{+}$(see figure 1 ). We will return to this point of view in section 3 .

In a realistic inflationary model, the de Sitter symmetries need to be broken. For this purpose, we introduce the dynamical inflaton field $\phi$. We give it a potential, $M_{\mathrm{pl}}^{2} \Lambda \rightarrow V(\phi)$, so that the field acquires a time-dependent expectation value $\phi=\bar{\phi}(t)$. This provides a natural clock measuring the time to the end of inflation. The expansion rate is now time dependent, $H \rightarrow H(t)$, and related to the evolution of the inflaton by the Einstein equations [28]

$$
\begin{aligned}
\dot{\bar{\phi}}^{2} & =-2 M_{\mathrm{pl}}^{2} \dot{H}, \\
V(\bar{\phi}) & =M_{\mathrm{pl}}^{2}\left(3 H^{2}+\dot{H}\right) .
\end{aligned}
$$




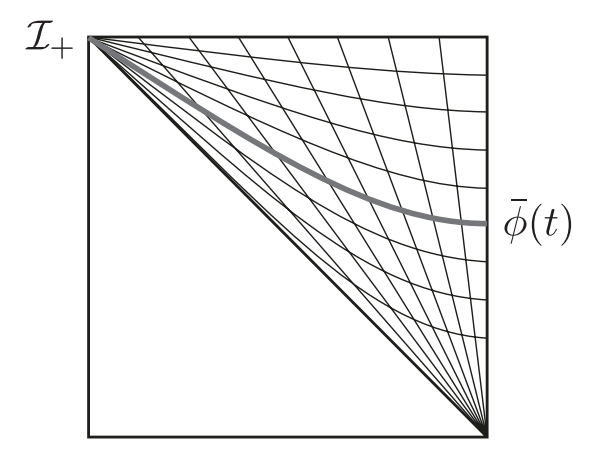

Figure 1. The time-dependent inflaton vev, $\bar{\phi}(t)$, introduces a preferred time slicing of de Sitter space.

The inflationary slow-roll parameter can then be written as

$$
\varepsilon=\frac{\frac{1}{2} \dot{\bar{\phi}}^{2}}{M_{\mathrm{pl}}^{2} H^{2}} .
$$

The size of $\varepsilon$ controls the breaking of the conformal symmetries of de Sitter, with the symmetries being restored in the limit $\varepsilon \rightarrow 0$.

We will assume that inflaton self-interactions are suppressed by a relatively large mass scale, $\Lambda^{2} \gg \dot{\bar{\phi}}$, while gravitational interactions are controlled by a lower scale, $\dot{\bar{\phi}}>M^{2} \gtrsim$ $H^{2}$. The hierarchy $\Lambda \gg M$ is protected because the gravitational coupling to the scalar sector is small. ${ }^{3}$ In that case, the leading breaking of the conformal symmetry comes from the inflaton potential, while higher-derivative interactions, like $(\partial \phi)^{4} / \Lambda^{4}$, will be suppressed $^{4}$ by powers of $\dot{\bar{\phi}} / \Lambda^{2} \ll H^{2} / M^{2}$. In addition, we may have functions of $\phi$ coupled to curvature tensors. These couplings were discussed systematically by Weinberg in [17]. Like Weinberg, we consider these terms to be perturbative corrections to the EinsteinHilbert action. This ensures that any ghost instabilities are moved outside the regime of validity of the effective theory. Using the field equations of the leading terms in the action, all inflaton-curvature couplings can be written in terms of couplings to the Weyl tensor

$$
W_{\mu \nu \rho \sigma} \equiv R_{\mu \nu \rho \sigma}-\frac{1}{2}\left(g_{\mu \rho} R_{\nu \sigma}-g_{\mu \sigma} R_{\nu \rho}-g_{\nu \rho} R_{\mu \sigma}+g_{\nu \sigma} R_{\mu \rho}\right)+\frac{R}{6}\left(g_{\mu \rho} g_{\nu \sigma}-g_{\nu \rho} g_{\mu \sigma}\right) .
$$

To study the tensor two-point function, we only need to consider the couplings to the square of the Weyl tensor,

$$
W^{2} \equiv W^{\mu \nu \rho \sigma} W_{\mu \nu \rho \sigma}=R^{\mu \nu \rho \sigma} R_{\mu \nu \rho \sigma}-2 R^{\mu \nu} R_{\mu \nu}+\frac{1}{3} R^{2},
$$

\footnotetext{
${ }^{3}$ To see this, consider, for instance, higher-curvature terms of the form $\Delta \mathcal{L} \equiv M_{\mathrm{pl}}^{2} R^{2} / M^{2}$. Using the leading-order equation of motion [17], $M_{\mathrm{pl}}^{2} R_{\mu \nu}=-\partial_{\mu} \phi \partial_{\nu} \phi-V(\phi) g_{\mu \nu}$, one finds $\Delta \mathcal{L} \subset(\partial \phi)^{4} / \Lambda^{4}$, with $\Lambda \equiv \sqrt{M_{\mathrm{pl}} M} \gg M$. We see that the effective cutoff of the scalar sector is enhanced by the large ratio $M_{\mathrm{pl}} / M$. We thank an anonymous referee for a discussion of this point.

${ }^{4}$ If the scale controlling inflaton interactions is smaller than $\dot{\bar{\phi}}$, then the power counting of the EFT changes significantly [29]. In this limit, inflaton fluctuations can propagate with a nontrivial sound speed, $c_{s} \ll 1$. In appendix $\mathrm{A}$, we show that a small sound speed induces a much stronger breaking of conformal symmetry than we wish to consider in this paper. Conversely, if we demand that the conformal symmetry is only broken by effects of order $\varepsilon$, then these symmetry-breaking operators have to be highly suppressed.
} 
and to the parity-violating term $W \widetilde{W} \equiv(\sqrt{-g})^{-1} \epsilon^{\mu \nu \rho \sigma} W_{\mu \nu}{ }^{\kappa \lambda} W_{\rho \sigma \kappa \lambda}$. Higher powers of the Weyl tensor will contribute to higher-point correlation functions, and are not relevant for the considerations of this paper. We will therefore study the following action

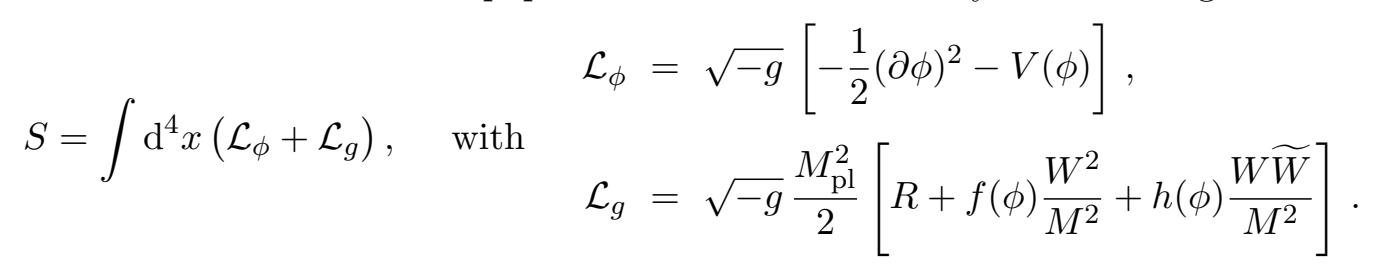

Notice that we have factored out the scale $M_{\mathrm{pl}}^{2}$ in $\mathcal{L}_{g}$. This is consistent with the structure expected in string effective actions [30], with $M$ playing the role of the string scale or the Kaluza-Klein scale. Since the Weyl tensor vanishes for any homogeneous FRW metric, the background slow-roll solution is still determined by the Einstein-Hilbert part of $\mathcal{L}_{g}$.

The effects of the parity-violating term $h(\phi) W \widetilde{W}$ have been studied in [31-34]. Since $W \widetilde{W}$ is a total derivative, this term vanishes if $h(\phi)$ is a constant. The correction to the tensor two-point function therefore comes from the field-dependent variation of $h(\phi)$. This leads to a difference in the amplitudes of the two chiralities of the tensor modes of order $\sqrt{\varepsilon} H^{2} / M^{2}$. In this paper, we will be interested in the effects of the coupling $f(\phi) W^{2}$. We will show that the field-dependent variation ${ }^{5}$ of $f(\phi)$ induces a sound speed for tensor fluctuations and a contribution to the tensor tilt of order $\sqrt{\varepsilon} H^{2} / M^{2}$.

Interestingly, the $f(\phi) W^{2}$ term in (2.10) is similar to a term in the effective action of the original Starobinsky model [38]. In these models, inflation is driven by a large number of conformally coupled fields whose stress tensor is induced by the conformal anomaly [39], $\left\langle T_{\mu}^{\mu}\right\rangle \supset c W^{2}$. The effective action that reproduces the conformal anomaly [40] includes the Weyl-squared term. It is not hard to imagine that variations of the model could contain a term of the form $f(\phi) W^{2}$. For example, such a term arises if one introduces the dilaton. It would be interesting to make this connection more precise [41]. ${ }^{6}$

\subsection{Violation of the consistency condition}

We now compute the scalar and tensor power spectra resulting from the action (2.10). We use the standard ADM decomposition of the metric [42]

$$
\mathrm{d} s^{2}=-N^{2} \mathrm{~d} t^{2}+g_{i j}\left(N^{i} \mathrm{~d} t+\mathrm{d} x^{i}\right)\left(N^{j} \mathrm{~d} t+\mathrm{d} x^{j}\right) .
$$

In comoving gauge, the inflaton is unperturbed, $\phi=\bar{\phi}(t)$, and the spatial metric can be written as

$$
g_{i j}=a^{2} e^{2 \zeta}\left(\delta_{i j}+\gamma_{i j}\right),
$$

where $\gamma_{i j}$ is a transverse and traceless tensor. At leading order, the curvature perturbation $\zeta$ and the tensor mode $\gamma_{i j}$ decouple and can be treated separately.

\footnotetext{
${ }^{5}$ For constant $f(\phi)$, the Weyl-squared term, $W^{2}$, can be put into the Gauss-Bonnet form, $R_{\mu \nu \rho \sigma}^{2}-$ $4 R_{\mu \nu}^{2}+R^{2}$, via the field redefinition $g_{\mu \nu} \rightarrow g_{\mu \nu}+f M_{\mathrm{pl}}^{-2}\left(-2 R_{\mu \nu}+\frac{5}{3} g_{\mu \nu} R\right)$ [35]. Since the Gauss-Bonnet term is a total derivative, it only contributes a boundary term. However, the field redefinition also changes the inflaton kinetic term and the normalization of the Einstein-Hilbert action, so the constant part of the function $f(\phi)$ is still physical. The phenomenology of inflationary models with a large Gauss-Bonnet term has been considered before in [36, 37].

${ }^{6}$ We thank Juan Maldacena for this suggestion.
} 
Scalars. We first consider the spectrum of the curvature perturbation $\zeta$. Since $W \widetilde{W}$ vanishes for scalar fluctuations, only $f(\phi) W^{2}$ contributes. At linear order in $\zeta$, the nonzero components of the Weyl tensor are $^{7}$

$$
\begin{aligned}
W_{0 j}^{0 i} & =\frac{1}{2}\left(\partial_{i} \partial_{j}-\frac{\delta_{i j}}{3} \nabla^{2}\right)\left(\frac{\varepsilon \zeta}{a^{2}}+\frac{1}{\nabla^{2}} \frac{d}{d t}(a \varepsilon \dot{\zeta})\right) \equiv F_{i j}, \\
W^{i j}{ }_{k l} & =\delta_{i k} F_{j l}+\delta_{j l} F_{i k}-\delta_{i l} F_{j k}-\delta_{j k} F_{i l},
\end{aligned}
$$

and hence $W^{2}=8 F_{i j}^{2}$. To eliminate terms with second-order time derivatives in the Weyl tensor, we use the leading-order equation of motion

$$
\ddot{\zeta}+\frac{d}{d t} \log \left(a^{3} \varepsilon\right) \dot{\zeta}-\frac{\nabla^{2}}{a^{2}} \zeta=0 .
$$

The quadratic action can then be written in the following form

$$
\frac{\mathcal{L}_{\zeta}}{M_{\mathrm{pl}}^{2}}=a^{3} \frac{\varepsilon}{c_{s}^{2}}\left(\dot{\zeta}^{2}-\frac{c_{s}^{2}}{a^{2}}(\vec{\nabla} \zeta)^{2}\right)
$$

where we have introduced the sound speed

$$
\frac{1}{c_{s}^{2}}-1 \equiv \frac{8}{3} \varepsilon f(\bar{\phi}) \frac{H^{2}}{M^{2}} .
$$

If we had kept the couplings of the inflaton to $R^{2}$ and $R_{\mu \nu}^{2}$, we would have found additional corrections to $c_{s}$ of the same order. Since the deviation from $c_{s}=1$ is suppressed by a factor of $\varepsilon \ll 1$, it will not play a significant role for the rest of this paper. For simplicity, we will therefore take $c_{s} \approx 1$, and write the power spectrum of $\zeta$ in the standard slow-roll form

$$
\Delta_{\zeta}^{2} \approx \frac{1}{8 \pi^{2}} \frac{1}{\varepsilon} \frac{H^{2}}{M_{\mathrm{pl}}^{2}},
$$

where the right-hand side is evaluated at horizon crossing, $k=a H$. We conclude that the coupling to the Weyl tensor has very little effect on the scalar power spectrum, and its main effect is a correction to the tensors.

Tensors. The linearized equation of motion for tensor fluctuations in Einstein gravity is

$$
\ddot{\gamma}_{i j}+3 H \dot{\gamma}_{i j}-\frac{\nabla^{2}}{a^{2}} \gamma_{i j}=0 .
$$

We will use this to simplify some of the perturbative corrections to the quadratic action for $\gamma$. At linear order in $\gamma$, the components of the Weyl tensor are

$$
\begin{aligned}
W^{0 i}{ }_{0 j} & =\frac{1}{4}\left(\ddot{\gamma}_{i j}+H \dot{\gamma}_{i j}+\frac{\nabla^{2}}{a^{2}} \gamma_{i j}\right), \\
W^{0 i}{ }_{j k} & =\frac{1}{2 a}\left(\dot{\gamma}_{i k, j}-\dot{\gamma}_{i j, k}\right),
\end{aligned}
$$

\footnotetext{
${ }^{7}$ To arrive at (2.13), we have used the linearized solutions to the Einstein constraint equations [23]: $\delta N=\dot{\zeta} / H$ and $\partial_{i} N^{i}=\varepsilon \dot{\zeta}-a^{-2} \nabla^{2} \zeta / H$.
} 


$$
\begin{aligned}
& W_{0 i}^{j k}=\frac{1}{2 a}\left(\dot{\gamma}_{i j, k}-\dot{\gamma}_{i k, j}\right), \\
& W_{k l}^{i j}=\frac{1}{2}\left\{\frac{1}{a^{2}}\left(\gamma_{i l, j k}+\gamma_{j k, i l}-\gamma_{i k, j l}-\gamma_{j l, i k}\right)\right. \\
&+\frac{1}{2}\left[\delta_{i l}\left(\ddot{\gamma}_{j k}+H \dot{\gamma}_{j k}-\frac{\nabla^{2}}{a^{2}} \gamma_{j k}\right)+\delta_{j k}\left(\ddot{\gamma}_{i l}+H \dot{\gamma}_{i l}-\frac{\nabla^{2}}{a^{2}} \gamma_{i l}\right)\right. \\
&\left.\left.\quad-\delta_{i k}\left(\ddot{\gamma}_{j l}+H \dot{\gamma}_{j l}-\frac{\nabla^{2}}{a^{2}} \gamma_{j l}\right)-\delta_{j l}\left(\ddot{\gamma}_{i k}+H \dot{\gamma}_{i k}-\frac{\nabla^{2}}{a^{2}} \gamma_{i k}\right)\right]\right\} .
\end{aligned}
$$

Substituting this into $W^{2}$, we get

$$
\begin{aligned}
W_{\rho \sigma}^{\mu \nu} W_{\mu \nu}^{\rho \sigma} & =4 W_{0 j}^{0 i} W_{0 i}^{0 j}+4 W^{0 i}{ }_{j k} W^{j k}{ }_{0 i}+W^{i j}{ }_{k l} W^{k l}{ }_{i j} \\
& =2\left(\ddot{\gamma}_{i j}+H \dot{\gamma}_{i j}+\frac{\nabla^{2}}{a^{2}} \gamma_{i j}\right)^{2}+4 \dot{\gamma}_{i j} \frac{\nabla^{2}}{a^{2}} \dot{\gamma}_{i j} .
\end{aligned}
$$

Using (2.18), this can be brought into the form of eq. (21) in [17]. After a few integrations by parts, we obtain

$$
\begin{aligned}
\frac{\mathcal{L}_{\gamma}}{M_{\mathrm{pl}}^{2}}= & \frac{a^{3}}{8} \frac{1}{c_{t}^{2}}\left(\dot{\gamma}_{i j}^{2}-\frac{c_{t}^{2}}{a^{2}}\left(\vec{\nabla} \gamma_{i j}\right)^{2}\right) \\
& -2 \frac{a^{3}}{M^{2}} f(\bar{\phi})\left[\gamma_{i j} \frac{\nabla^{2}}{a^{2}}\left(\ddot{\gamma}_{i j}+3 H \dot{\gamma}_{i j}-\frac{\nabla^{2}}{a^{2}} \gamma_{i j}\right)\right] \\
& -2 \frac{a^{3}}{M^{2}} \frac{d f(\bar{\phi})}{d t} \gamma_{i j} \frac{\nabla^{2}}{a^{2}} \dot{\gamma}_{i j}-\frac{4}{M^{2}} \frac{d h(\bar{\phi})}{d t} \epsilon^{i j k 0} \gamma_{i l} \partial_{j} \nabla^{2} \gamma_{k l} .
\end{aligned}
$$

The second line in (2.21) vanishes after using the equation of motion (2.18) once more. The last line is proportional to $\dot{\bar{\phi}}$ and hence is suppressed in the slow-roll limit. ${ }^{8}$ This leaves the first line, which is the quadratic action for tensors with a nontrivial sound speed

$$
\frac{1}{c_{t}^{2}}-1 \equiv 8 f(\bar{\phi}) \frac{H^{2}}{M^{2}}
$$

In [26], it was shown that a tensor sound speed can always be set to unity by a disformal transformation [27], followed by a Weyl rescaling to take the action to Einstein frame. These two metric transformations trade the nontrivial tensor sound speed for a scalar sound speed, $\tilde{c}_{s}$, and a modified Hubble rate, $\tilde{H}(\tilde{t})$. In the new frame, the tensor spectrum takes the standard form, $\Delta_{\gamma}^{2} \propto \tilde{H}^{2} / M_{\mathrm{pl}}^{2}$, but the scalar spectrum is modified. Of course, predictions for observables are frame-independent [26, 43], so the choice of frame is simply a matter of convenience. In particular, the violation of the consistency condition that we will find is a frame-independent conclusion. ${ }^{9}$ We leave the details to appendix B, but one point is worth emphasizing here. The violation of the consistency condition in the new

\footnotetext{
${ }^{8}$ The last term in (2.21), although slow-roll suppressed, is phenomenologically interesting because it leads to chiral gravitational waves [31-34]. Incidentally, the size of the chiral splitting, $\sqrt{\varepsilon} H^{2} / M^{2}$, is of the same order as the correction to the tensor tilt that we will get from the rest of the action.

${ }^{9}$ We thank Paolo Creminelli and Filippo Vernizzi for a discussion of these issues.
} 
frame is not the same as that found in $P(X)$-theories [44]. Rather, it is the time derivative of $\tilde{c}_{s}$ that modifies the tensor-to-scalar ratio.

The tensor sound speed leads to a simple rescaling of the standard tensor power spectrum. Summing over the two graviton polarizations, we obtain

$$
\Delta_{\gamma}^{2}=\frac{2}{\pi^{2}} \frac{H^{2}}{M_{\mathrm{pl}}^{2}} \frac{1}{c_{t}},
$$

where the right-hand side is evaluated at $c_{t} k=a H$. If the inflaton-Weyl coupling is a small correction to the leading gravitational action - as we are assuming in order to avoid propagating ghost degrees of freedom - then $c_{t}$ can't deviate much from unity. The main effect is not the size of $c_{t}$, but its time dependence. ${ }^{10}$ In particular, the tensor-to-scalar ratio approximately still takes the form predicted by standard slow-roll inflation

$$
r \equiv \frac{\Delta_{\gamma}^{2}}{\Delta_{\zeta}^{2}}=\frac{16 \varepsilon}{c_{t}} \approx 16 \varepsilon
$$

However, the tensor tilt can still receive an important correction due to the time dependence of $c_{t}$. Crucially, the evolution of $c_{t}(t)$ is coupled to that of $\bar{\phi}(t)$. This will induce a tilt of the tensor spectrum proportional to $\dot{\bar{\phi}} \propto \sqrt{\varepsilon}$. To see this, let us define a slow-variation parameter for the tensor sound speed, $\varepsilon_{t}$, and express it in terms of the slow-roll parameter $\varepsilon$ :

$$
\varepsilon_{t} \equiv \frac{\dot{c}_{t}}{H c_{t}}=\mp 4 c_{t}^{2} b \sqrt{2 \varepsilon} \frac{H^{2}}{M^{2}}+\left(1-c_{t}^{2}\right) \varepsilon,
$$

where $b \equiv M_{\mathrm{pl}} f^{\prime}$ is a dimensionless constant. Taking the scale of variation of $f(\phi)$ to be of order $\Lambda$, we get $b \sim M_{\mathrm{pl}} / \Lambda$, which may be large if $\Lambda \ll M_{\mathrm{pl}}$. The fractional change of (2.23) per Hubble time then determines the tensor tilt

$$
n_{t} \equiv \frac{d \ln \Delta_{\gamma}^{2}}{d \ln k}=-2 \varepsilon-\varepsilon_{t} \approx-2 \varepsilon \pm 4 b \sqrt{2 \varepsilon} \frac{H^{2}}{M^{2}},
$$

where we have ignored a small shift in the coefficient of the standard contribution, $-2 \varepsilon$. Notice that the correction has undetermined sign, so it seems to allow a blue tilt for the tensor spectrum, even without a violation of the null energy condition (NEC). ${ }^{11}$ The second term in (2.26) leads to a modification of the tensor consistency condition

$$
-\frac{8 n_{t}}{r}=1 \mp \frac{4 b}{\sqrt{2 \varepsilon}} \frac{H^{2}}{M^{2}} .
$$

We see that the violation of the relation $n_{t}=-r / 8$ is enhanced for small $\varepsilon$ (and large $b$ ), but suppressed by $H^{2} / M^{2}$. In the stringy regime of inflation, $H^{2} / M^{2}$ can be of order one ${ }^{12}$ and our proposed modification of the tensor spectrum could be a significant effect.

\footnotetext{
${ }^{10}$ The fact that $c_{t}$ is never allowed to deviate too far from unity puts a constraint on the time dependence of $c_{t}$, and hence on the function $f(\phi)$ in $(2.22)$.

${ }^{11}$ In the Einstein frame, with $\tilde{c}_{t}=1$, a blue tilt still corresponds to a violation of the NEC, $\dot{\tilde{H}}>0$, but without inducing the gradient instability that this usually implies [26, 45].

${ }^{12}$ When $M$ is the string scale, the ratio $H / M$ is constrained by the fact that we require the Hagedorn temperature to remain above the de Sitter temperature in order to avoid a phase transition of the system [46].
} 
Testing the tensor consistency condition observationally is challenging (see [47, 48] for a recent discussion). Naturally, the observational prospects improve for large tensor-toscalar ratio and if a large range of scales can be accessed (maybe with futuristic direct detection experiments [49-51]). A blue tensor spectrum would be easier to detect.

\section{CFT interpretation}

The freeze-out of quantum fluctuations during inflation allows us to recast cosmological expectation values in terms of the 'wavefunction of the universe', $\Psi[\zeta, \gamma]$. This wavefunction computes late-time expectation values of superhorizon fluctuations. The isometries of de Sitter space imply that the coefficients of the wavefunction can be interpreted as correlation functions of the stress tensor in a putative conformal field theory $[22,23]$. The small breaking of conformal symmetry during inflation is modelled as a small deformation of the CFT, which can be treated perturbatively [24]. In this section, we will show that this alternative point of view reproduces the results of the previous section.

\subsection{Wavefunction of the universe}

The wavefunction of the universe can be computed by a saddle-point approximation, $\Psi \approx e^{i S_{c l}}$, where the action $S_{c l}$ is evaluated for a classical solution with certain Dirichlet boundary conditions [23]. The result takes the following form

$$
\Psi=e^{i S_{\mathrm{div}}} e^{W_{0}[\zeta, \gamma]}, \quad \text { with } \quad W_{0}=\frac{1}{2} \int \mathrm{d}^{3} k\left(\zeta_{\vec{k}} \zeta_{-\vec{k}}\left\langle T_{\vec{k}} T_{-\vec{k}}\right\rangle^{\prime}+\sum_{s} \gamma_{\vec{k}}^{s} \gamma_{-\vec{k}}^{s}\left\langle T_{\vec{k}}^{s} T_{-\vec{k}}^{s}\right\rangle^{\prime}\right)
$$

The local divergent piece, $e^{i S_{\text {div }}}$, is a pure phase factor, and thus drops out of expectation values. The coefficient functions $\left\langle T_{\vec{k}} T_{-\vec{k}}\right\rangle^{\prime}$ and $\left\langle T_{\vec{k}}^{s} T_{-\vec{k}}^{s}\right\rangle^{\prime}$ may be interpreted as the correlation functions of the trace and the trace-free part of the stress tensor $T_{i j}$ of a dual field theory $[22,23,52] .{ }^{13}$ The power spectra of $\zeta$ and $\gamma$ are then computed by a simple Gaussian integration

$$
\begin{aligned}
\left\langle\zeta_{\vec{k}} \zeta_{-\vec{k}}\right\rangle^{\prime} & =\int \mathcal{D} \zeta \zeta_{\vec{k}} \zeta_{-\vec{k}}|\Psi[\zeta]|^{2}=-\frac{1}{2 \operatorname{Re}\left\langle T_{\vec{k}} T_{-\vec{k}}\right\rangle^{\prime}} \\
\left\langle\gamma_{\vec{k}}^{s} \gamma_{-\vec{k}}^{s}\right\rangle^{\prime} & =\int \mathcal{D} \gamma^{s} \gamma_{\vec{k}}^{s} \gamma_{-\vec{k}}^{s}\left|\Psi\left[\gamma^{s}\right]\right|^{2}=-\frac{1}{2 \operatorname{Re}\left\langle T_{\vec{k}}^{s} T_{-\vec{k}}^{s}\right\rangle^{\prime}}
\end{aligned}
$$

The diffeomorphism invariance of gravitational theories implies that the generators of coordinate transformations act as constraints on the wavefunction [53]. These constraint equations are the conformal Ward identities of the coefficient functions $\left\langle T_{\vec{k}} T_{-\vec{k}}\right\rangle^{\prime}$ and $\left\langle T_{\vec{k}}^{s} T_{-\vec{k}}^{s}\right\rangle^{\prime}$. In a CFT, these constraints imply

$$
\left\langle T_{\vec{k}} T_{-\vec{k}}\right\rangle^{\prime}=0, \quad\left\langle T_{\vec{k}}^{s} T_{-\vec{k}}^{s}\right\rangle^{\prime}=c_{T} k^{3},
$$

\footnotetext{
${ }^{13}$ The CFT that describes the de Sitter cosmology is not unitary and has some unusual features, mostly related to the spectrum of the dimensions of operators. Our analysis will only use the mapping of the symmetries between the bulk and the boundary, and does not rely on a deeper meaning of dS/CFT.
} 
where $c_{T}$ is the central charge. We see that there are no $\zeta$-fluctuations and the gravitational sector consists only of gravitons. In terms of bulk quantities, the central charge is

$$
c_{T}=-\frac{1}{4} \frac{M_{\mathrm{pl}}^{2}}{H^{2}} .
$$

In a quasi-de Sitter background, with finite slow-roll parameter $\varepsilon$, some of the conformal symmetries are softly broken (see appendix A). The effects of this weak symmetry breaking can be treated perturbatively.

\subsection{Conformal perturbation theory}

A suitable framework for analyzing field theories that are almost conformal is conformal perturbation theory [24]. We now wish to show that such an analysis reproduces the results of section 2 .

We deform the CFT by a local primary operator $[54]^{14}$

$$
S=S_{\mathrm{CFT}}+\varphi \int \mathrm{d}^{3} z \mathcal{O}(\vec{z})
$$

where $\varphi$ is a small coupling. ${ }^{15}$ We take the perturbing operator to be marginally relevant, so its dimension is $\Delta \equiv 3-\lambda$, with $0<\lambda \ll 1$. The small expansion parameter dual to $\sqrt{\varepsilon}$ will be a combination of $\varphi$ and $\lambda$. For convenience, we normalize the two-point function of $\mathcal{O}$ by the central charge

$$
\left\langle\mathcal{O}_{\vec{k}} \mathcal{O}_{-\vec{k}}\right\rangle^{\prime}=c_{T} k^{3-2 \lambda} .
$$

For small $\varphi$, the two-point function of the stress tensor can be computed perturbatively as

$$
\begin{aligned}
\left\langle T_{i j} T_{k l}\right\rangle= & \left\langle T_{i j} T_{k l} e^{-\varphi \int \mathrm{d}^{3} z \mathcal{O}}\right\rangle_{0} \\
=\left\langle T_{i j} T_{k l}\right\rangle_{0} & -\varphi \int \mathrm{d}^{3} z\left\langle T_{i j} T_{k l} \mathcal{O}(\vec{z})\right\rangle_{0} \\
& \quad+\frac{\varphi^{2}}{2} \int \mathrm{d}^{3} z \mathrm{~d}^{3} w\left\langle T_{i j} T_{k l} \mathcal{O}(\vec{z}) \mathcal{O}(\vec{w})\right\rangle_{0}+\cdots,
\end{aligned}
$$

where the expectation values $\left\langle T_{i j} T_{k l} \ldots \mathcal{O}\right\rangle_{0}$ are computed using the CFT operator algebra, and in general are constrained by Ward identities. We will use the following trace Ward identities obeyed by the stress tensor

$$
\begin{gathered}
\left\langle T^{i}{ }_{i}(\vec{x}) T_{k l}(\vec{y}) \mathcal{O}(\vec{z})\right\rangle_{0}=\lambda \delta(\vec{x}-\vec{z})\left\langle T_{k l}(\vec{y}) \mathcal{O}(\vec{z})\right\rangle_{0}=0, \\
\left\langle T^{i}{ }_{i}(\vec{x}) T_{k l}(\vec{y}) \mathcal{O}(\vec{z}) \mathcal{O}(\vec{w})\right\rangle_{0}=\lambda\left[\delta(\vec{x}-\vec{z})\left\langle T_{k l}(\vec{y}) \mathcal{O}(\vec{z}) \mathcal{O}(\vec{w})\right\rangle_{0}+(\vec{z} \leftrightarrow \vec{w})\right],
\end{gathered}
$$

\footnotetext{
${ }^{14}$ Of course, CFTs are characterized by a set of correlation functions rather than by an action. Here, $S_{\mathrm{CFT}}$ is simply a metaphoric way of characterizing the content of the original CFT. In practice, calculations in conformal perturbation theory are always performed at the level of correlation functions.

${ }^{15}$ In conformal perturbation theory, one usually tunes the perturbation so that the beta function vanishes and the theory flows to a new conformal fixed point [24]. Since we are mainly interested in the parametric scaling of the corrections, we will not perform this additional step and thus we do not worry about the particular renormalization group flow.
} 


$$
\left\langle T^{i}{ }_{i}(\vec{x}) T^{k}{ }_{k}(\vec{y}) \mathcal{O}(\vec{z}) \mathcal{O}(\vec{w})\right\rangle_{0}=\lambda^{2}\left[\delta(\vec{x}-\vec{z}) \delta(\vec{y}-\vec{w})\langle\mathcal{O}(\vec{z}) \mathcal{O}(\vec{w})\rangle_{0}+(\vec{z} \leftrightarrow \vec{w})\right]
$$

where, in the last identity, we have dropped an irrelevant contact term, with support when $\vec{x}=\vec{y}$. In a reparametrization invariant theory, $\nabla^{i}\left\langle T_{i j}\right\rangle=0$, we furthermore have $k^{i}\left\langle T_{i j}(\vec{k}) T_{k l}(-\vec{k})\right\rangle^{\prime}=0$. Imposing this constraint implies that $\left\langle T_{i j} T_{k l}\right\rangle$ has the following form

$$
\left\langle T_{i j}(\vec{k}) T_{k l}(-\vec{k})\right\rangle^{\prime}=\frac{1}{4}\left[\delta_{i j}^{\perp} \delta_{k l}^{\perp}\left\langle T_{\vec{k}} T_{-\vec{k}}\right\rangle^{\prime}+\left(\delta_{i k}^{\perp} \delta_{j l}^{\perp}+\delta_{i l}^{\perp} \delta_{j k}^{\perp}-\delta_{i j}^{\perp} \delta_{k l}^{\perp}\right)\left\langle T_{\vec{k}}^{s} T_{-\vec{k}}^{s}\right\rangle^{\prime}\right]
$$

where $\delta_{i j}^{\perp} \equiv \delta_{i j}-k_{i} k_{j} / k^{2}$.

In general, $\left\langle T_{\vec{k}}^{s} T_{-\vec{k}}^{s}\right\rangle^{\prime}$ and $\left\langle T_{\vec{k}} T_{-\vec{k}}\right\rangle^{\prime}$ are arbitrary functions of $k$, but in a theory with approximate conformal symmetry, we expect them to be approximately scale invariant. The breaking of scale invariance can be studied in powers of $\varphi$ :

- First, let us look at the two-point function of the trace, $\left\langle T_{\vec{k}} T_{-\vec{k}}\right\rangle$. It follows from the trace Ward identity (3.9) that this vanishes at order $\varphi$. The scalar two-point function is therefore only generated at order $\varphi^{2}$. Using (3.10) and (3.11), we find

$$
\left\langle T_{\vec{k}} T_{-\vec{k}}\right\rangle^{\prime}=\varphi^{2} \lambda^{2}\left\langle\mathcal{O}_{\vec{k}} \mathcal{O}_{-\vec{k}}\right\rangle^{\prime}=c_{T} \varphi^{2} \lambda^{2} k^{3-2 \lambda} .
$$

The tensor-to-scalar ratio therefore is

$$
r \equiv \frac{\left\langle T_{\vec{k}} T_{-\vec{k}}\right\rangle^{\prime}}{\left\langle T_{\vec{k}}^{s} T_{-\vec{k}}^{s}\right\rangle^{\prime}}=\varphi^{2} \lambda^{2} .
$$

Comparing this to the bulk result, $r=16 \varepsilon$, we identify the following duality map: $\varphi \lambda \leftrightarrow \pm 4 \sqrt{\varepsilon}$.

- Next, we consider the correction to $\left\langle T_{\vec{k}}^{s} T_{-\vec{k}}^{s}\right\rangle^{\prime}$. At $O(\varphi)$, we require the integral of the three-point function $\left\langle T^{s} T^{s} \mathcal{O}\right\rangle_{0}$. In position space, we have

$$
\left\langle T_{i j}(\vec{x}) T_{k l}(\vec{y}) \mathcal{O}(\vec{z})\right\rangle_{0}^{\prime}=c_{T} f_{T T \mathcal{O}} \mathcal{T}_{i j k l}(\vec{x}-\vec{y}, \vec{y}-\vec{z}, \vec{z}-\vec{x}),
$$

where an explicit expression for the tensor structure $\mathcal{T}_{i j k l}$ can be found in the classic work of Osborn and Petkou [55], cf. eqs. (3.3)-(3.6). We have identified $c_{T} f_{T T \mathcal{O}}$ with the coefficient $a$ of eq. (3.4) in [55]. Integrating (3.15) over $\vec{z}$, and transforming to momentum space, we get

$$
\left\langle T_{\vec{k}}^{s} T_{-\vec{k}}^{s} \mathcal{O}(\overrightarrow{0})\right\rangle_{0}^{\prime}=c_{T} \alpha(\lambda) f_{T T \mathcal{O}} k^{3-\lambda}
$$

where $\alpha(\lambda)$ is a numerical coefficient. We have confirmed by explicit integration that $\alpha(\lambda)$ is finite, even in the limit $\lambda \rightarrow 0 .{ }^{16}$ This implies that $T_{i j}$ doesn't need to be renormalized, and also means that perturbing the CFT by an exactly marginal

\footnotetext{
${ }^{16}$ We also found that $\alpha(\lambda)$ vanishes for a two-dimensional CFT. This is to be expected from the $c$ theorem in two dimensions. It is also consistent with our bulk interpretation, since the Weyl tensor vanishes identically in three dimensions.
} 
operator simply shifts the coefficient of the stress tensor two-point function. Substituting (3.16) into (3.8), we get

$$
\left\langle T_{\vec{k}}^{s} T_{-\vec{k}}^{s}\right\rangle^{\prime}=c_{T} k^{3}\left(1-n_{t} \ln k+\cdots\right),
$$

where we have dropped a small $O(\varphi)$ shift of the amplitude, and defined

$$
n_{t} \equiv-\varphi \lambda \alpha(\lambda) f_{T T \mathcal{O}}
$$

This reproduces the $O(\sqrt{\varepsilon})$ contribution in the bulk result (2.26), if we make the following identification: $\alpha(\lambda) f_{T T \mathcal{O}} \leftrightarrow \sqrt{2} b H^{2} / M^{2}$. The $O\left(\varphi^{2}\right)$ term in the $\left\langle T_{\vec{k}}^{s} T_{-\vec{k}}^{s}\right\rangle$ correlator contains the standard tensor tilt proportional to $\varepsilon$. This contribution depends on the details of the CFT and its various OPE coefficients.

The main result of this section was the confirmation that a tensor tilt is generated at $O(\varphi)$, while the tensor-to-scalar ratio is only non-zero at $O\left(\varphi^{2}\right)$. The tensor tilt comes from a specific three-point function, whose size is set by $f_{T T \mathcal{O}}$ in the boundary CFT, and by $H^{2} / M^{2}$ in the bulk action. The standard result of Einstein gravity is recovered for $f_{T T \mathcal{O}} \rightarrow 0$, or $M \rightarrow \infty$.

\section{Conclusions}

The weak breaking of conformal symmetry during inflation can be used to constrain the predictions for cosmological correlators - e.g. [1, 2, 5]. This is especially relevant in inflationary models in which the scale suppressing higher-curvature corrections is close to the Hubble scale. In this paper, we have studied the coupling of the inflaton field to highercurvature tensors in models with a minimal breaking of conformal symmetry. We showed that the most general correction to the tensor two-point function is captured by a coupling to the square of the Weyl tensor. This interaction modifies the consistency condition of single-field slow-roll inflation

$$
-\frac{8 n_{t}}{r}=1 \mp \frac{4 b}{\sqrt{2 \varepsilon}} \frac{H^{2}}{M^{2}}
$$

The correction can have either sign, and may dominate over the prediction from Einstein gravity if $H / M$ is not too small. We consider this an interesting signature of highercurvature corrections during inflation.

We have left a few open questions for future work:

- What is the precise connection between our effective action (2.10) and the original Starobinsky model [38]? Both models rely on softly broken conformal invariance, and the effective actions even contain some terms of the same functional form. Making this relationship more precise would be very interesting [41].

- How naturally does our scenario arise in explicit string compactifications? Under which circumstances is a weakly broken conformal symmetry maintained in the fourdimensional effective theory? Is there a relation to conformal supergravity [56-58]? 
- If a violation of the tensor consistency condition were to be observed, how would we convince ourselves that it comes from higher-curvature effects? In particular, it is well-known that a violation of $r=-8 n_{t}$ can also arise from modifications of the scalar spectrum in models with a nontrivial scalar sound speed [44] and/or isocurvature fluctuations [59]. However, in that case we also expect strong interactions in the scalar sector, which can be tested for through measurements of primordial non-Gaussianity. In contrast, in our proposal we do not predict a strong counterpart in scalar nonGaussianity. A positive test of our scenario would be looking for correlated signatures of a low string scale, such as angular dependence in the scalar bispectrum [2] and specific forms of tensor non-Gaussianity [1].

- Can we get blue tensors? Our analysis determines neither the sign of the coupling to the Weyl tensor, nor its time dependence. While the sign may be constrained by requiring tensors to propagate subluminally, we see no a priori way to constrain the rate of change of the coupling. At the moment, blue tensors therefore seem to be a legitimate possibility.

\section{Acknowledgments}

We thank Mustafa Amin, David Berenstein, Paolo Creminelli, Garrett Goon, Liam McAllister, Paul McFadden, Mehrdad Mirbabayi, Jorge Noreña, Rafael Porto, Jan Pieter van der Schaar, Ilya Shapiro, Stephen Shenker, Filippo Vernizzi, and especially Juan Maldacena and Hugh Osborn for helpful discussions. We thank Juan Maldacena, Liam McAllister and Mehrdad Mirbabayi for comments on a draft. G.P. thanks the KITP for hospitality during the program 'Quantum Gravity Foundations: UV to IR'. Research at the KITP is supported in part by the National Science Foundation under Grant No. NSF PHY1125915. This paper has the KITP preprint number NSF-KITP-15-125. D.B. and G.P. thank the Aspen Center for Physics (NSF Grant 1066293) for their hospitality while this work was being completed. D.B., H.L. and G.P. acknowledge support from a Starting Grant of the European Research Council (ERC STG Grant 279617). H.L. is supported by the Cambridge Overseas Trust and the William Georgetti Scholarship.

\section{A Comments on conformal symmetry}

In this appendix, we study the breaking of conformal symmetry in an inflationary spacetime. By conformal symmetry, we mean the action of the de Sitter isometries on quantum fields in the background geometry. We will show that dilatations and special conformal transformations are broken by an amount controlled by $\varepsilon=-\dot{H} / H^{2}$. Moreover, we will demonstrate that in theories with nontrivial sound speed, $c_{s} \ll 1$, special conformal symmetry is broken even in the limit $\varepsilon \rightarrow 0$.

- First, let us consider a massless scalar field $f$ in pure de Sitter space

$$
S_{d S}=\frac{1}{2} \int \mathrm{d}^{4} x \frac{\left(f^{\prime}\right)^{2}-(\vec{\nabla} f)^{2}}{(H \eta)^{2}} .
$$


Conformal transformations act as follows

$$
\begin{aligned}
\delta_{\lambda} f & =\lambda\left(\eta f^{\prime}+\vec{x} \cdot \vec{\nabla} f\right), \\
\delta_{b} f & =2 \vec{b} \cdot \vec{x}\left(\eta f^{\prime}+\vec{x} \cdot \vec{\nabla} f\right)+\left(\eta^{2}-x^{2}\right) \vec{b} \cdot \vec{\nabla} f .
\end{aligned}
$$

It is straightforward to check that the action (A.1) is invariant under these transformations.

- Next, let us repeat the analysis for an inflationary background. The action of a massless scalar in quasi-de Sitter space is

$$
S_{I}=\frac{1}{2} \int \mathrm{d}^{4} x a^{2}(\eta)\left(\left(f^{\prime}\right)^{2}-(\vec{\nabla} f)^{2}\right) \equiv \int \mathrm{d}^{4} x \mathcal{L}_{I}, \quad a(\eta)=-\frac{1}{H \eta(1-\varepsilon)} .
$$

After integrations by parts, we obtain the following variations of the action

$$
\begin{aligned}
& \delta_{\lambda} S_{I}=2 \lambda \int \mathrm{d}^{4} x \varepsilon \mathcal{L}_{I}, \\
& \delta_{b} S_{I}=2 \int \mathrm{d}^{4} x(\vec{b} \cdot \vec{x}) \varepsilon \mathcal{L}_{I} .
\end{aligned}
$$

As advertised, dilatations and SCTs are broken by an amount proportional to $\varepsilon$.

- Finally, we consider a massless scalar field with a nontrivial speed of sound

$$
S_{c_{s}}=\frac{1}{2} \int \mathrm{d}^{4} x \frac{1}{c_{s}^{2}} \frac{\left(f^{\prime}\right)^{2}-c_{s}^{2}(\vec{\nabla} f)^{2}}{(H \eta)^{2}} .
$$

Assuming $c_{s}=$ const., for simplicity, the variation of the action gives

$$
\begin{aligned}
& \delta_{\lambda} S_{c_{s}}=0 \\
& \delta_{b} S_{c_{s}}=2 \int \mathrm{d}^{4} x \frac{1-c_{s}^{2}}{c_{s}^{2}} \frac{\eta f^{\prime}(\vec{b} \cdot \vec{\nabla}) f}{(H \eta)^{2}} .
\end{aligned}
$$

We see that scale invariance is retained, while special conformal invariance is broken. For time-dependent $c_{s}(t)$, dilatations would be broken as well.

\section{B Comments on Einstein frame}

In [26], it was shown that a nontrivial tensor sound speed can be set to unity by a disformal transformation [27]. This is followed by a conformal transformation, which brings the action back to Einstein frame. The combined transformation is given by

$$
g_{\mu \nu} \rightarrow c_{t}^{-1}\left[g_{\mu \nu}+\left(1-c_{t}^{2}\right) n_{\mu} n_{\nu}\right]
$$

where $n_{\mu} \propto \partial_{\mu} \phi$ is the unit vector orthogonal to the constant-time hypersurfaces. The action in the new frame then has a trivial sound speed for tensors, $\tilde{c}_{t}=1$, but a nontrivial sound speed for scalars, $\tilde{c}_{s}=c_{t}^{-1}$. In this appendix, we show that observables are the 
same in both frames. In particular, we will find that the modification to the consistency condition (2.27) is still present in the new frame.

Consider the action (2.10) in comoving gauge. At quadratic order in fluctuations and at leading order in slow-roll, we get

$$
S=\frac{M_{\mathrm{pl}}^{2}}{8} \int \mathrm{d} t \mathrm{~d}^{3} x\left(\mathcal{L}_{\zeta}+\mathcal{L}_{\gamma}\right), \quad \text { with } \quad \begin{aligned}
\mathcal{L}_{\zeta} & =8 a^{3} \varepsilon\left[\dot{\zeta}^{2}-a^{-2}(\vec{\nabla} \zeta)^{2}\right] \\
\mathcal{L}_{\gamma} & =a^{3} c_{t}^{-2}\left[\dot{\gamma}_{i j}^{2}-a^{-2} c_{t}^{2}\left(\vec{\nabla} \gamma_{i j}\right)^{2}\right] .
\end{aligned}
$$

After performing the transformation (B.1), the background line element becomes $\mathrm{d} s^{2}=$ $-c_{t} \mathrm{~d} t^{2}+c_{t}^{-1} a^{2} \mathrm{~d} \vec{x}^{2}$. Rescaling the time and the scale factor,

$$
\mathrm{d} \tilde{t}=c_{t}^{1 / 2}(t) \mathrm{d} t, \quad \tilde{a}(\tilde{t})=c_{t}^{-1 / 2}(t) a(t),
$$

we get $\mathrm{d} s^{2}=-\mathrm{d} \tilde{t}^{2}+\tilde{a}^{2} \mathrm{~d} \vec{x}^{2}$. The curvature perturbation $\zeta$ and the tensor fluctuations $\gamma_{i j}$ transform as spacetime scalars, so the action (B.2) takes the form

$$
S=\frac{M_{\mathrm{pl}}^{2}}{8} \int \mathrm{d} \tilde{t} \mathrm{~d}^{3} x\left(\tilde{\mathcal{L}}_{\tilde{\zeta}}+\tilde{\mathcal{L}}_{\tilde{\gamma}}\right), \quad \text { with } \quad \begin{aligned}
& \tilde{\mathcal{L}}_{\tilde{\zeta}}=8 \tilde{a}^{3} \varepsilon\left[c_{t}^{2}\left(\partial_{\tilde{t}} \tilde{\zeta}\right)^{2}-\tilde{a}^{-2}(\vec{\nabla} \tilde{\zeta})^{2}\right], \\
& \tilde{\mathcal{L}}_{\tilde{\gamma}}=\tilde{a}^{3}\left[\left(\partial_{\tilde{t}} \tilde{\gamma}_{i j}\right)^{2}-\tilde{a}^{-2}\left(\vec{\nabla} \tilde{\gamma}_{i j}\right)^{2}\right] .
\end{aligned}
$$

Hence, in the new frame, the tensors propagate with a trivial sound speed, $\tilde{c}_{t}=1$, but the scalars have a modified sound speed, $\tilde{c}_{s}=c_{t}^{-1}$. Notice that $c_{t}<1$ implies $\tilde{c}_{s}>1$. It is not unusual that a non-local field redefinition maps a purely luminal theory to one with apparent superluminality (e.g. [60-62]). In such a situation, the presence of a superluminal mode does not imply a violation of relativistic causality. ${ }^{17}$

At leading order in slow-roll, the tensor power spectrum takes the standard form

$$
\Delta_{\gamma}^{2}=\frac{2}{\pi^{2}} \frac{\tilde{H}^{2}}{M_{\mathrm{pl}}^{2}}
$$

in terms of the new Hubble parameter $\tilde{H} \equiv \partial_{\tilde{t}} \ln \tilde{a} \approx c_{t}^{-1 / 2} H$. The tensor tilt is hence also of the usual form, $n_{t}=-2 \tilde{\varepsilon}$, and all nontrivial features have been moved to the scalar sector. The power spectrum of curvature perturbations is

$$
\Delta_{\zeta}^{2}=\frac{1}{8 \pi^{2}} \frac{1}{\varepsilon \tilde{c}_{s}} \frac{\tilde{H}^{2}}{M_{\mathrm{pl}}^{2}}
$$

where

$$
\varepsilon=\tilde{\varepsilon}+\frac{1}{2} \tilde{\varepsilon}_{s}, \quad \text { with } \quad \tilde{\varepsilon}_{s}=-\varepsilon_{t} .
$$

If we neglect the small shift in the amplitude due to $\tilde{c}_{s} \approx 1$, then the tensor-to-scalar ratio is

$$
r \approx 16\left[\tilde{\varepsilon}+\frac{1}{2} \tilde{\varepsilon}_{s}\right]
$$

\footnotetext{
${ }^{17}$ We thank Paolo Creminelli for explaining this to us.
} 
Hence, although the tensor tilt is standard in the new frame, the tensor-to-scalar ratio now is non-standard. The tensor consistency condition is then given by

$$
-\frac{8 n_{t}}{r}=\frac{\tilde{\varepsilon}}{\tilde{\varepsilon}+\frac{1}{2} \tilde{\varepsilon}_{s}}\left(=1+\frac{1}{2} \frac{\varepsilon_{t}}{\varepsilon}\right) .
$$

We see that the consistency condition is still modified in the new frame, but now the effect is coming from the time dependence of a nontrivial scalar sound speed, $\tilde{\varepsilon}_{s} \neq 1$. Substituting $\tilde{\varepsilon}$ and $\tilde{\varepsilon}_{s}$ in terms of the parameters in the original frame, $\varepsilon$ and $\varepsilon_{t}$, we find complete agreement with our previous result (2.27).

Open Access. This article is distributed under the terms of the Creative Commons Attribution License (CC-BY 4.0), which permits any use, distribution and reproduction in any medium, provided the original author(s) and source are credited.

\section{References}

[1] J.M. Maldacena and G.L. Pimentel, On graviton non-Gaussianities during inflation, JHEP 09 (2011) 045 [arXiv: 1104.2846] [inSPIRE].

[2] N. Arkani-Hamed and J. Maldacena, Cosmological collider physics, arXiv:1503.08043 [INSPIRE].

[3] I. Antoniadis, P.O. Mazur and E. Mottola, Conformal invariance, dark energy and CMB non-Gaussianity, JCAP 09 (2012) 024 [arXiv: 1103.4164] [INSPIRE].

[4] P. Creminelli, Conformal invariance of scalar perturbations in inflation, Phys. Rev. D 85 (2012) 041302 [arXiv:1108.0874] [INSPIRE].

[5] I. Mata, S. Raju and S. Trivedi, CMB from CFT, JHEP 07 (2013) 015 [arXiv:1211.5482] [INSPIRE].

[6] A. Ghosh, N. Kundu, S. Raju and S.P. Trivedi, Conformal invariance and the four point scalar correlator in slow-roll inflation, JHEP 07 (2014) 011 [arXiv:1401.1426] [INSPIRE].

[7] N. Kundu, A. Shukla and S.P. Trivedi, Constraints from conformal symmetry on the three point scalar correlator in inflation, JHEP 04 (2015) 061 [arXiv:1410.2606] [INSPIRE].

[8] P. McFadden and K. Skenderis, Holography for cosmology, Phys. Rev. D 81 (2010) 021301 [arXiv: 0907.5542] [INSPIRE].

[9] P. McFadden and K. Skenderis, Holographic non-Gaussianity, JCAP 05 (2011) 013 [arXiv: 1011.0452] [INSPIRE].

[10] P. McFadden and K. Skenderis, Cosmological 3-point correlators from holography, JCAP 06 (2011) 030 [arXiv: 1104.3894] [INSPIRE].

[11] A. Bzowski, P. McFadden and K. Skenderis, Holographic predictions for cosmological 3-point functions, JHEP 03 (2012) 091 [arXiv: 1112.1967] [INSPIRE].

[12] A. Bzowski, P. McFadden and K. Skenderis, Holography for inflation using conformal perturbation theory, JHEP 04 (2013) 047 [arXiv: 1211.4550] [INSPIRE].

[13] P. McFadden, On the power spectrum of inflationary cosmologies dual to a deformed CFT, JHEP 10 (2013) 071 [arXiv:1308.0331] [INSPIRE]. 
[14] N. Kundu, A. Shukla and S.P. Trivedi, Ward identities for scale and special conformal transformations in inflation, arXiv:1507.06017 [INSPIRE].

[15] J.E. Lidsey et al., Reconstructing the inflation potential: an overview, Rev. Mod. Phys. 69 (1997) 373 [astro-ph/9508078] [INSPIRE].

[16] N. Kaloper, M. Kleban, A.E. Lawrence and S. Shenker, Signatures of short distance physics in the cosmic microwave background, Phys. Rev. D 66 (2002) 123510 [hep-th/0201158] [INSPIRE].

[17] S. Weinberg, Effective field theory for inflation, Phys. Rev. D 77 (2008) 123541 [arXiv: 0804.4291] [INSPIRE].

[18] B. Zwiebach, Curvature squared terms and string theories, Phys. Lett. B 156 (1985) 315 [INSPIRE].

[19] M. Brigante, H. Liu, R.C. Myers, S. Shenker and S. Yaida, Viscosity bound violation in higher derivative gravity, Phys. Rev. D 77 (2008) 126006 [arXiv:0712.0805] [INSPIRE].

[20] Y. Kats and P. Petrov, Effect of curvature squared corrections in AdS on the viscosity of the dual gauge theory, JHEP 01 (2009) 044 [arXiv:0712.0743] [INSPIRE].

[21] A. Buchel, R.C. Myers and A. Sinha, Beyond $\eta / s=1 / 4 \pi$, JHEP 03 (2009) 084 [arXiv: 0812.2521] [INSPIRE].

[22] A. Strominger, The dS/CFT correspondence, JHEP 10 (2001) 034 [hep-th/0106113] [INSPIRE].

[23] J.M. Maldacena, Non-Gaussian features of primordial fluctuations in single field inflationary models, JHEP 05 (2003) 013 [astro-ph/0210603] [INSPIRE].

[24] A.B. Zamolodchikov, Renormalization group and perturbation theory near fixed points in two-dimensional field theory, Sov. J. Nucl. Phys. 46 (1987) 1090 [Yad. Fiz. 46 (1987) 1819] [INSPIRE].

[25] F. Larsen and R. McNees, Inflation and de Sitter holography, JHEP 07 (2003) 051 [hep-th/0307026] [INSPIRE].

[26] P. Creminelli, J. Gleyzes, J. Noreña and F. Vernizzi, Resilience of the standard predictions for primordial tensor modes, Phys. Rev. Lett. 113 (2014) 231301 [arXiv:1407.8439] [INSPIRE].

[27] J.D. Bekenstein, The relation between physical and gravitational geometry, Phys. Rev. D 48 (1993) 3641 [gr-qc/9211017] [INSPIRE].

[28] D. Baumann, TASI lectures on inflation, arXiv:0907.5424 [INSPIRE].

[29] C. Cheung, P. Creminelli, A.L. Fitzpatrick, J. Kaplan and L. Senatore, The effective field theory of inflation, JHEP 03 (2008) 014 [arXiv: 0709.0293] [INSPIRE].

[30] D. Baumann and L. McAllister, Inflation and string theory, arXiv:1404.2601 [INSPIRE].

[31] A. Lue, L.-M. Wang and M. Kamionkowski, Cosmological signature of new parity violating interactions, Phys. Rev. Lett. 83 (1999) 1506 [astro-ph/9812088] [INSPIRE].

[32] S. Alexander and J. Martin, Birefringent gravitational waves and the consistency check of inflation, Phys. Rev. D 71 (2005) 063526 [hep-th/0410230] [INSPIRE].

[33] C.R. Contaldi, J. Magueijo and L. Smolin, Anomalous CMB polarization and gravitational chirality, Phys. Rev. Lett. 101 (2008) 141101 [arXiv:0806.3082] [InSPIRE]. 
[34] T. Takahashi and J. Soda, Chiral primordial gravitational waves from a Lifshitz point, Phys. Rev. Lett. 102 (2009) 231301 [arXiv:0904.0554] [INSPIRE].

[35] S. Deser and A.N. Redlich, String induced gravity and ghost freedom, Phys. Lett. B 176 (1986) 350 [Erratum ibid. B 186 (1987) 461] [INSPIRE].

[36] S. Tsujikawa and M. Sami, String-inspired cosmology: late time transition from scaling matter era to dark energy universe caused by a Gauss-Bonnet coupling, JCAP 01 (2007) 006 [hep-th/0608178] [INSPIRE].

[37] M. Satoh and J. Soda, Higher curvature corrections to primordial fluctuations in slow-roll inflation, JCAP 09 (2008) 019 [arXiv: 0806.4594] [INSPIRE].

[38] A.A. Starobinsky, A new type of isotropic cosmological models without singularity, Phys. Lett. B 91 (1980) 99 [INSPIRE].

[39] M.J. Duff, Twenty years of the Weyl anomaly, Class. Quant. Grav. 11 (1994) 1387 [hep-th/9308075] [INSPIRE].

[40] R.J. Riegert, A nonlocal action for the trace anomaly, Phys. Lett. B 134 (1984) 56 [INSPIRE].

[41] D. Baumann et al., Fluctuations in anomaly-induced inflation, work in progress.

[42] R.L. Arnowitt, S. Deser and C.W. Misner, The dynamics of general relativity, Gen. Rel. Grav. 40 (2008) 1997 [gr-qc/0405109] [INSPIRE].

[43] S. Tsujikawa, Disformal invariance of cosmological perturbations in a generalized class of Horndeski theories, JCAP 04 (2015) 043 [arXiv: 1412.6210] [INSPIRE].

[44] X. Chen, M.-X. Huang, S. Kachru and G. Shiu, Observational signatures and non-Gaussianities of general single field inflation, JCAP 01 (2007) 002 [hep-th/0605045] [INSPIRE].

[45] P. Creminelli, M.A. Luty, A. Nicolis and L. Senatore, Starting the universe: stable violation of the null energy condition and non-standard cosmologies, JHEP 12 (2006) 080 [hep-th/0606090] [INSPIRE].

[46] J.J. Atick and E. Witten, The Hagedorn transition and the number of degrees of freedom of string theory, Nucl. Phys. B 310 (1988) 291 [INSPIRE].

[47] J. Caligiuri and A. Kosowsky, Inflationary tensor perturbations after BICEP2, Phys. Rev. Lett. 112 (2014) 191302 [arXiv: 1403.5324] [INSPIRE].

[48] S. Dodelson, How much can we learn about the physics of inflation?, Phys. Rev. Lett. 112 (2014) 191301 [arXiv:1403.6310] [INSPIRE].

[49] T.L. Smith, M. Kamionkowski and A. Cooray, Direct detection of the inflationary gravitational wave background, Phys. Rev. D 73 (2006) 023504 [astro-ph/0506422] [INSPIRE].

[50] T.L. Smith, H.V. Peiris and A. Cooray, Deciphering inflation with gravitational waves: cosmic microwave background polarization vs. direct detection with laser interferometers, Phys. Rev. D 73 (2006) 123503 [astro-ph/0602137] [INSPIRE].

[51] S. Chongchitnan and G. Efstathiou, Prospects for direct detection of primordial gravitational waves, Phys. Rev. D 73 (2006) 083511 [astro-ph/0602594] [INSPIRE].

[52] E. Witten, Quantum gravity in de Sitter space, hep-th/0106109 [INSPIRE]. 
[53] B.S. DeWitt, Quantum theory of gravity 1. The canonical theory, Phys. Rev. 160 (1967) 1113 [INSPIRE].

[54] J.P. van der Schaar, Inflationary perturbations from deformed CFT, JHEP 01 (2004) 070 [hep-th/0307271] [INSPIRE].

[55] H. Osborn and A.C. Petkou, Implications of conformal invariance in field theories for general dimensions, Annals Phys. 231 (1994) 311 [hep-th/9307010] [INSPIRE].

[56] E.S. Fradkin and A.A. Tseytlin, Conformal supergravity, Phys. Rept. 119 (1985) 233 [INSPIRE].

[57] N. Berkovits and E. Witten, Conformal supergravity in twistor-string theory, JHEP 08 (2004) 009 [hep-th/0406051] [INSPIRE].

[58] J. Maldacena, Einstein gravity from conformal gravity, arXiv:1105.5632 [INSPIRE].

[59] D. Wands, N. Bartolo, S. Matarrese and A. Riotto, An observational test of two-field inflation, Phys. Rev. D 66 (2002) 043520 [astro-ph/0205253] [InSPIRE].

[60] P. Creminelli, M. Serone and E. Trincherini, Non-linear representations of the conformal group and mapping of Galileons, JHEP 10 (2013) 040 [arXiv:1306.2946] [INSPIRE].

[61] C. de Rham, M. Fasiello and A.J. Tolley, Galileon duality, Phys. Lett. B 733 (2014) 46 [arXiv: 1308.2702] [INSPIRE].

[62] C. De Rham, L. Keltner and A.J. Tolley, Generalized Galileon duality, Phys. Rev. D 90 (2014) 024050 [arXiv: 1403.3690] [INSPIRE]. 